\title{
Series of Low-Grade B-Cell Lymphoma: Follicular Lymphoma, Marginal Zone Lymphoma, Waldenstrom Macroglobulinemia and Mantle Cell Lymphoma
}

\author{
Ganesh Kasinathan \\ Department of Hematology, Ampang Hospital, Selangor, Malaysia \\ Email: ganeshkasinathan11@hotmail.com
}

How to cite this paper: Kasinathan, G. (2020) Series of Low-Grade B-Cell Lymphoma: Follicular Lymphoma, Marginal Zone Lymphoma, Waldenstrom Macroglobulinemia and Mantle Cell Lymphoma. Open Access Library Journal, 7: e6759.

https://doi.org/10.4236/oalib.1106759

Received: August 28, 2020

Accepted: September 20, 2020

Published: September 23, 2020

Copyright (ङ 2020 by author(s) and Open Access Library Inc.

This work is licensed under the Creative Commons Attribution International License (CC BY 4.0).

http://creativecommons.org/licenses/by/4.0/

\begin{abstract}
Low grade B-cell non-Hodgkin lymphoma (LBNHL) is usually slow growing and most often incurable. Case 1: A 47-year-old Malay gentleman was diagnosed with relapsed Stage IV Grade 2 Follicular Lymphoma (Follicular lymphoma international prognostic index, FLIPI 2: intermediate risk). He was initially treated with 6 cycles of standard rituximab, cyclophosphamide, doxorubicin, vincristine and prednisolone (R-CHOP) polychemotherapy at first presentation and was in remission for 8 years until he relapsed with new inguinal lymphadenopathy and bone marrow involvement. He was re-treated with 6 cycles of rituximab-bendamustine (R-B), in which he is in complete remission for the past 2 years. Case 2: A 45-year old Malay female presented with previously untreated Stage IV extranodal marginal zone lymphoma of the left pleura with nodal and bone marrow involvement which did not respond adequately to 3 cycles of R-CHOP chemotherapy. Her unilateral pleural effusion was persistent, and she required re-treatment with 4 cycles R-B immunochemotherapy. She is now in complete remission for the past 18 months. Case 3: A 70-year old Malay gentleman was diagnosed with Waldenstrom macroglobulinemia without hyperviscosity syndrome. He was treated with 2 cycles of R-B immunochemotherapy. However, he developed worsening anemia. He was re-treated with 6 cycles of Bortezomib-Rituximab-Dexamethasone (B-R-D) immunochemotherapy, in which he is currently in complete remission for the past 16 months. Case 4: A 59-year-old Malay gentleman diagnosed with Stage IV non-TP53 mutated leukemic phase mantle cell lymphoma achieved complete remission after completing 6 cycles of intensive chemotherapy followed by consolidation autologous stem cell transplant.
\end{abstract}




\section{Subject Areas}

Hematology

\section{Keywords}

Low-Grade B-Cell Lymphoma, Bone Marrow Involvement, Rituximab, Pleural Effusion

\section{Introduction}

Low grade B-cell non-Hodgkin lymphoma (LBNHL) also known as indolent lymphoma is usually slow growing and most often incurable. The classic prototypes of LBNHL are: CD5-CD10-marginal zone lymphoma, lymphoplasmacytic lymphoma/waldenstrom macroglobulinemia and hairy cell leukemia, CD5-CD10+ mantle cell lymphoma and small lymphocytic lymphoma/chronic lymphocytic leukemia, and CD5-CD10+ follicular lymphoma [1]. LGBNL is more commonly seen in males and older individuals between the age of 50 and 64. Follicular lymphoma (FL) is the most common of all low-grade lymphomas and represents $20 \%$ of all non-hodgkin lymphomas [2]. The common three subtypes of FL include in-situ follicular neoplasia, duodenal-type FL and paediatric-type FL. The World health organisation (WHO) 2016 classifies FL into three main grades based on the population of centroblasts. The median survival of low-grade FL (Grade 1, Grade 2, and Grade 3A) commonly exceeds 10 years with a rate of histologic transformation of $2 \%-3 \%$ per year [3].

Marginal zone lymphoma (MZL) is a heterogenous disorder which arises from malignant transformation of marginal zone B-cells. WHO 2016 classifies MZL into three main types: extranodal MZL (mucosa associated lymphoid tissue-MALT lymphoma), splenic MZL (SMZL) and nodal MZL (NMZL). Extranodal MZL affects most frequently the stomach, ocular adnexae, conjunctivae, salivary glands, thyroid, skin, small intestines and lungs [4]. Hashimotos thyroiditis and Sjogen syndrome are risk factors of MALT lymphoma of the thyroid and salivary glands respectively. NMZL and SMZL may involve the bone marrow in one-third to half of the patients. Nodal MZL usually carries a worse prognosis than MALT lymphoma and is often disseminated at diagnosis involving cervical, abdominal and inguinal lymph nodes and may demonstrate Ig $\mathrm{M}$ paraproteinemia in $10 \%$ of patients [5]. Paediatric-type nodal MZL which is more common in boys enjoys a much better prognosis than adult NMZL. On the other hand, SMZL presents with symptomatic splenomegaly, bone marrow and peripheral blood involvement (peripheral lymphocytosis). Some of them may manifest features of warm autoimmune hemolytric anemia (AIHA), immune thrombocytopenia (ITP), cold agglutinin disease, angioedema and chronic hepatitis- $\mathrm{C}$ infection. The angioedema is thought to occur due to $\mathrm{C} 1$ esterase inhibitor deficiency. The onset of SMZL may be preceded by clonal B-cell lymphocy- 
tosis of marginal zone origin (CBL-MZ)-presence of B-lymphocytes in the peripheral blood [6].

Waldenstrom macroglobulinemia (WM) is more commonly seen in the elderly Caucasians with a median survival of 5 years and may be associated with organomegaly (hepatomegaly, splenomegaly), lymphadenopathy and bone marrow involvement. Lymphoplasmacytic lymphoma (LPL) which does meet the criteria for diagnosis of WM may express other immunoglobulins such as IgA or IgG instead of IgM. WM is often preceded by IgM monoclonal gammopathy of undetermined significance (IgM MGUS) or smoldering WM and may progress to multiple myeloma, large cell lymphoma or infrequently amyloidosis [7]. Higher levels of $\operatorname{IgM}(>30 \mathrm{~g} / \mathrm{L}$ ) causes hyperviscosity complications such as hemorrhage, headache, epistaxis, stroke and altered consciousness.

Mantle cell lymphoma (MCL) is more commonly seen in older males and most patients follow an aggressive course. There are a few subtypes of MCL; leukemic non-nodal MCL (an indolent MCL which presents with bone marrow involvement and splenomegaly without lymphadenopathy), pleomorphic, blastoid and chronic lymphocytic leukemic (CLL) variants. MCL has a higher propensity to relapse as compared to other low-grade lymphomas.

Hairy cell leukemia and chronic lymphocytic leukemia/small lymphocytic lymphoma are not described in this series.

\section{Case Presentation 1}

A 47-year-old gentleman of Malay ethnicity presented to the hematology unit of a peripheral hospital with a two-month history of cervical lymph node swellings and lethargy. Physical examination revealed bilateral cervical and axillary lymphadenopathy. A cervical lymph node excision biopsy demonstrated effaced lymph node architecture and presence of abnormal lymphoid follicles comprising of 12 - 13 centroblasts per high power field microscopy consistent with Grade 2 follicular lymphoma. Pan-computed tomography (CT) imaging showed Stage IIB disease with the largest lymph node measuring $5 \times 5 \mathrm{~cm}$. He did not have any bone marrow involvement. He was treated with 6 cycles of RCHOP polychemotherapy. He was in complete remission (CR) for 8 years until he developed new small inguinal lymph node swellings of $3 \times 3 \mathrm{~cm}$ and peripheral cytopenia. However, he had no B symptoms and was decided for watchful waiting. He then developed constitutive symptoms with enlarging inguinal lymph node swelling a year later. His complete blood count (CBC) showed anemia (hemoglobin: $8.1 \mathrm{~g} / \mathrm{dL}$, normal range: $13.5-16.5 \mathrm{~g} / \mathrm{dL}$ ), leukopenia (total white cell count: $2.1 \times 10^{9} / \mathrm{L}$, normal range: $4-10 \times 10^{9} / \mathrm{L}$ ) and thrombocytopenia (platelet: $85 \times 10^{9} / \mathrm{L}$, normal range: $150-400 \times 10^{9} / \mathrm{L}$ ). He did not have any raised lactate dehydrogenase or serum Beta-2-microglobulin levels. The peripheral blood film did not portray any abnormal lymphocytes. The inguinal lymph node excision biopsy (Figures 1(a)-(f)) was consistent with Grade 2 FL. A Pan-CT imaging demonstrated more than 3 groups of enlarged lymph nodes 


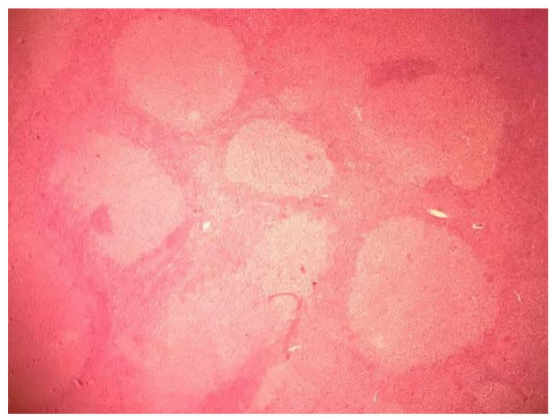

(a)

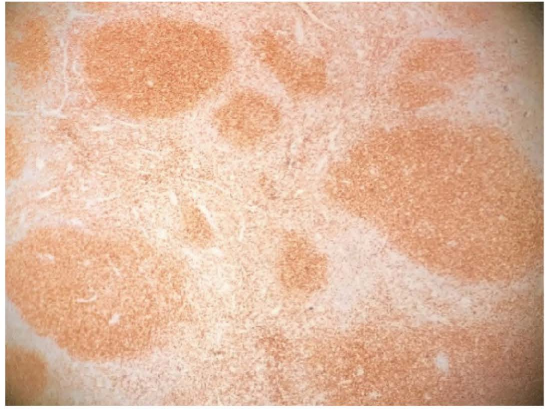

(c)

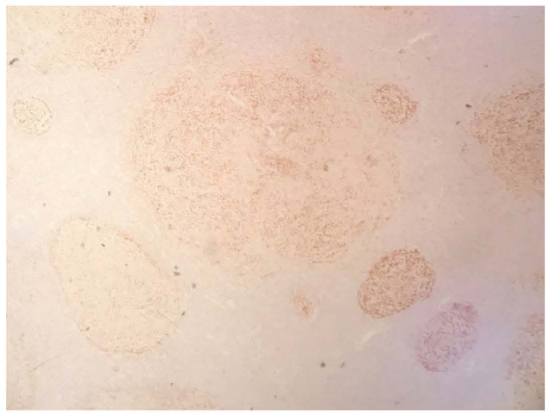

(e)

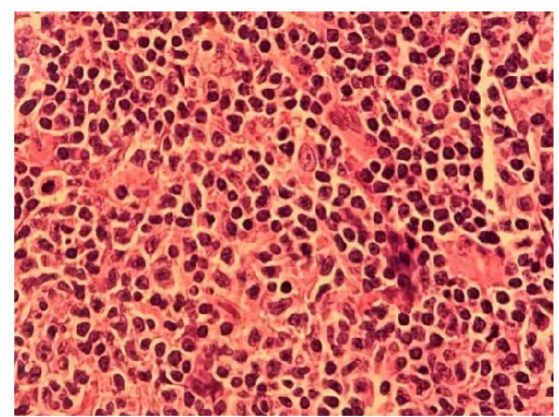

(b)

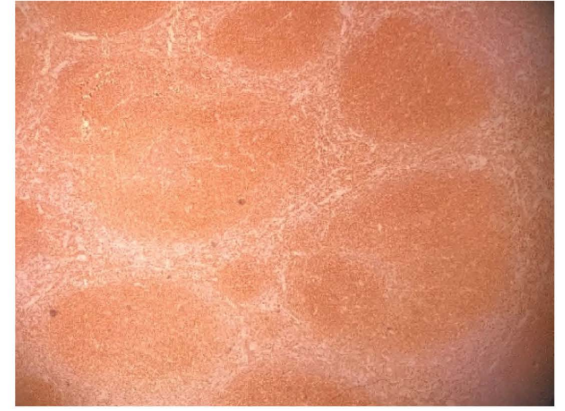

(d)

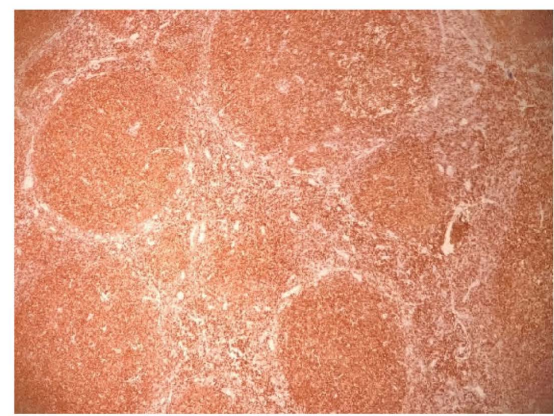

(f)

Figure 1. (a) Inguinal lymph node biopsy H\&E low power shows numerous abnormal lymphoid follicles (b) Inguinal lymph node biopsy H\&E high power shows 12 - 13 centroblasts per high power field microscopy ((c)-(f)) Panel of immunohistochemical staining (Dako Artisan Link) shows neoplastic cells staining positive for CD10, CD20, CD21 (follicular dendritic meshwork) and BCL-2.

above and below the diaphragm, the largest measuring $5 \times 6 \mathrm{~cm}$ with hepatosplenomegaly. His bone marrow aspiration showed numerous cleaved lymphocytes resembling buttock cells. The trephine biopsy demonstrated abnormal paratrabecular lymphoid follicles with the neoplastic cells staining positive for CD10, CD19, CD20, CD21, CD79a, BCL2 and BCL6. He had a Groupe d'Etude des Lymphomes Folliculaires (GELF) score of 3 (presence of 3 and more nodal sites, each with diameter of $>3 \mathrm{~cm}$, B symptoms, cytopenia). He was diagnosed as relapsed Stage IV Grade 2 FL (FLIPI-2: intermediate risk). He was treated with 6 cycles of Rituximab-Bendamustine (R-B) immunochemotherapy every 28 days. In view of his low-grade disease, he was not subjected to an autologous stem cell transplant. He is now in complete remission for the past 2 years. 


\section{Case Presentation 2}

A 45-year old lady of Malay ethnicity presented to the medical department of a periphery hospital with a 6-week history of bilateral inguinal lymph node swelling and intermittent dyspnoea. She also complained of loss of weight and anorexia. She did not have any significant family history. She was a non-smoker, a teetotaler and previously worked as an office administrator. Physical examination revealed a mildly tachypnoeic female with a respiratory rate of 24 breaths per minute. She had palpable bilateral inguinal lymphadenopathy, the largest measuring $3 \times 4 \mathrm{~cm}$ and a spleen of $4 \mathrm{~cm}$ below the left costal margin. She had no other organomegaly. A complete blood count showed a hemoglobin of 8.4 $\mathrm{g} / \mathrm{dL}$, total white blood cell count of $15 \times 10^{9} / \mathrm{L}$ and a platelet count of $130 \times$ $10^{9} /$ L. Peripheral blood film (Figure $2(\mathrm{a})$ ) revealed non-villous abnormal lymphocytes. The chest radiograph demonstrated a massive left sided pleural effusion. Pleurocentesis was performed with 2 litres of exudative straw-coloured fluid drained. An inguinal lymph node excision biopsy demonstrated sheets of small-to-medium abnormal lymphocytes exhibiting irregular nuclei and abundant cytoplasm. The neoplastic cells stained positive for CD19, CD20, CD22, CD79a, BCL2 and lambda light chain. They were negative for CD5, CD10, MUM1 and BCL6. These findings were consistent with marginal zone lymphoma. She was then referred to the tertiary hematology referral centre. Her left sided pleural effusion recurred. A diagnostic pleuroscopy was performed with the pleural biopsy being consistent with extranodal marginal zone lymphoma. A pan-CT imaging showed diffuse lymphadenopathy affecting bilateral cervical, axillary and inguinal lymph nodes, the largest measuring $3 \times 3 \mathrm{~cm}$. Bone marrow aspiration (Figure 2(b)) portrayed numerous abnormal non-villous small-tomedium sized lymphocytes with reduced trilineage haematopoiesis. The bone marrow trephine biopsy (Figures 2(c)-(f)) showed presence of infiltration by clusters of abnormal lymphocytes which expressed positivity for CD20, CD79a and BCL2. They were negative for CD5, CD10, MUM1 and BCL6. Her serum protein electrophoresis, immunofixation and free light chain revealed elevated IgG paraproteinemia of $12 \mathrm{~g} / \mathrm{L}$ with lambda light chain of $890 \mathrm{~g} / \mathrm{L}$. She was diagnosed as Stage IV extranodal marginal zone lymphoma of the left pleura with nodal and marrow involvement (MALT-IPI: intermediate risk). She was treated with 3 cycles of R-CHOP polychemotherapy. However, her pleural effusion persisted. A chemical talc pleurodesis was performed. She was re-treated with 4 cycles of R-B chemotherapy, in which she responded. She is now in complete remission for the past 18 months.

\section{Case Presentation 3}

A 70-year-old male of Malay ethnicity who had no previous medical illness presented to the department of hematology with fever, lethargy, anorexia and loss of weight for the past 4 weeks. He did not exhibit symptoms of hyperviscosity. $\mathrm{He}$ had no significant family history. He was a chronic smoker, a teetotaller and did 


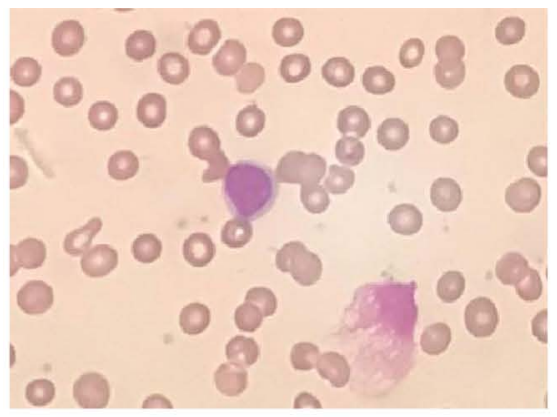

(a)

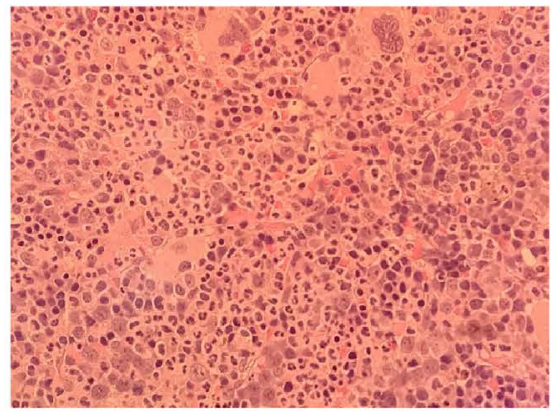

(c)

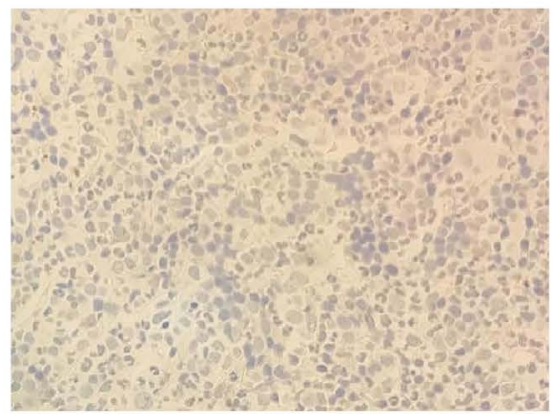

(e)

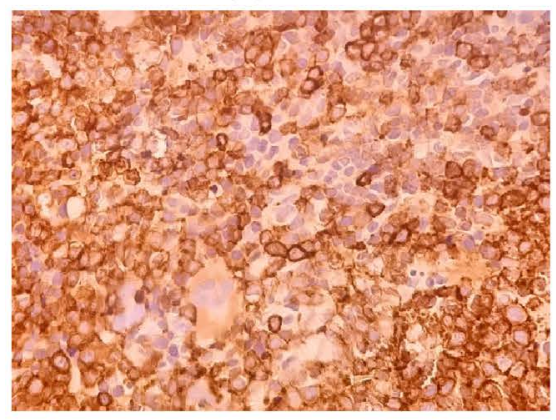

(g)

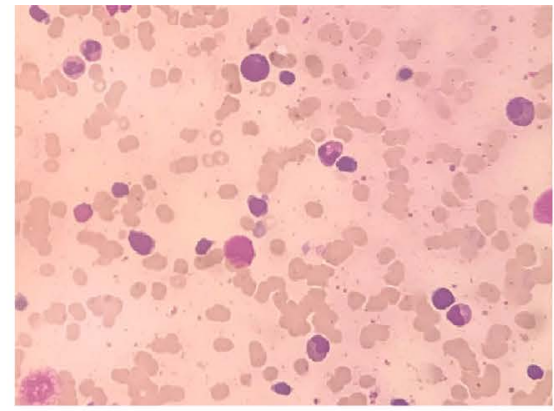

(b)

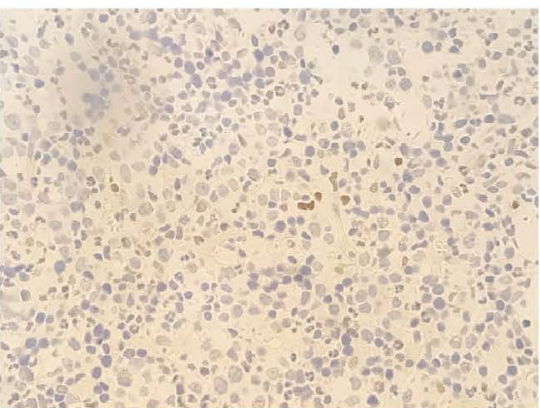

(d)

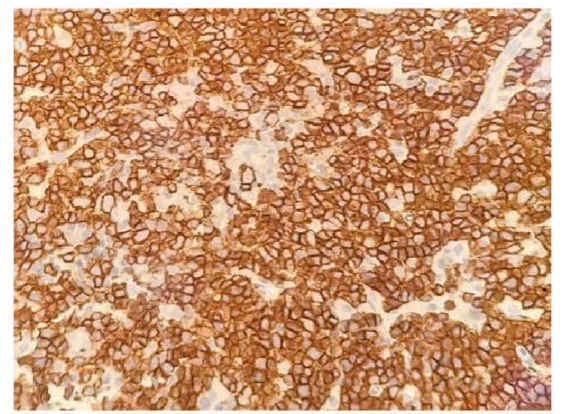

(f)

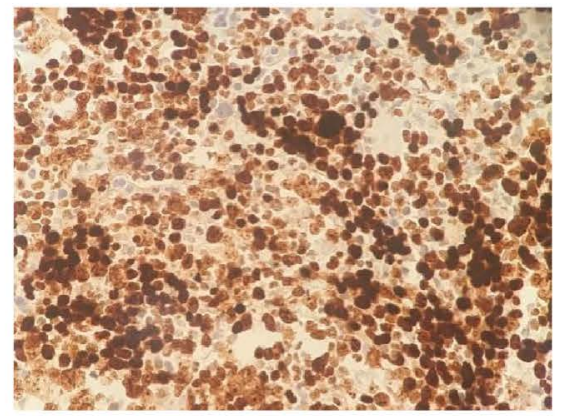

(h)

Figure 2. (a) Peripheral blood film shows non-villous abnormal lymphocytes. (b) Bone marrow aspiration demonstrates lymphocytosis with suppression of trilineage haematopoiesis (c) Bone marrow trephine biopsy H\&E shows infiltration by clusters of abnormal lymphocytes ((d)-(g)) Immunohistochemical staining (Dako Artisan Link) of trephine biopsy shows neoplastic cells stain negative for CD5 and CD10. Neoplastic cells stain positively for CD20 and CD43. (h) Ki 67 (proliferation index) is $40 \%$.

not consume recreational drugs. He worked previously as a bank clerk.

Physical examination revealed an alert gentleman with a blood pressure of 
$110 / 70$ and a pulse rate of 92 beats per minute. There were no cutaneous lesions. He did not exhibit any lymphadenopathy or organomegaly. Other systemic examinations were unremarkable.

Complete blood count showed a hemoglobin of $8.2 \mathrm{~g} / \mathrm{dL}$, total white cell count of $120 \times 10^{9} / \mathrm{L}$, absolute lymphocyte count of $110 \times 10^{9} / \mathrm{L}$ and platelet count of $85 \times 10^{9} / \mathrm{L}$. His lactate dehydrogenase $(\mathrm{LDH})$, serum beta- 2 microglobulin, serum calcium, renal and liver function parameters were all within the normal range.

The peripheral blood film showed anemia, numerous lymphoplasmacytoid cells and true thrombocytopenia. The bone marrow aspiration revealed many lymphoplasmacytoid cells with suppressed trilineage haematopoiesis. The trephine biopsy (Figures 3(a)-(f)) revealed infiltration by sheets of lymphoplasmacytoid cells. The neoplastic cells were lambda restricted and stained positive

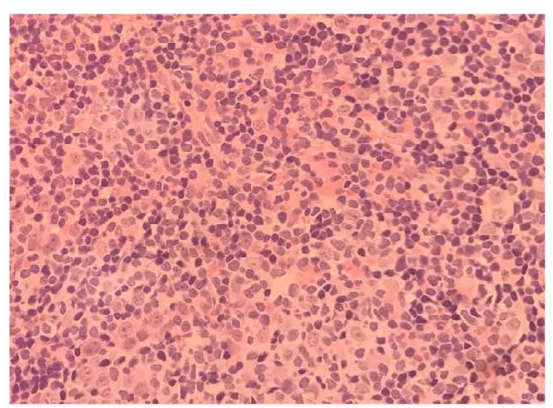

(a)

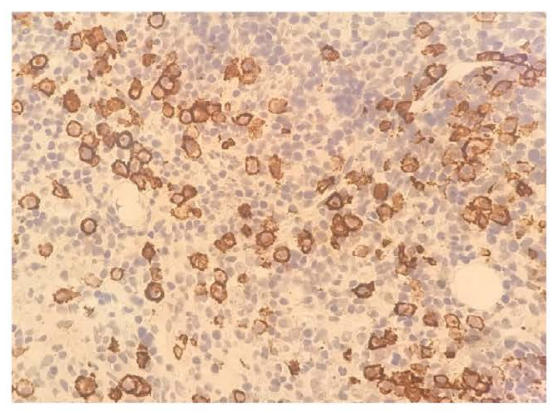

(c)

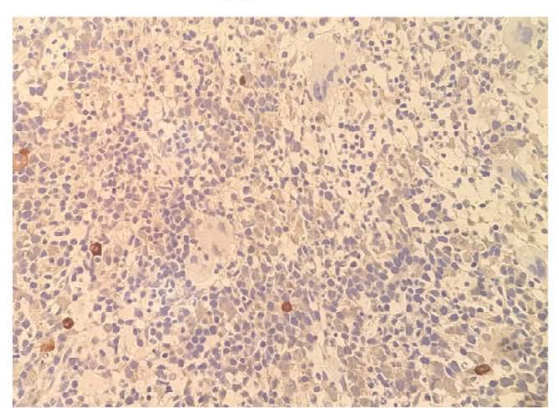

(e)

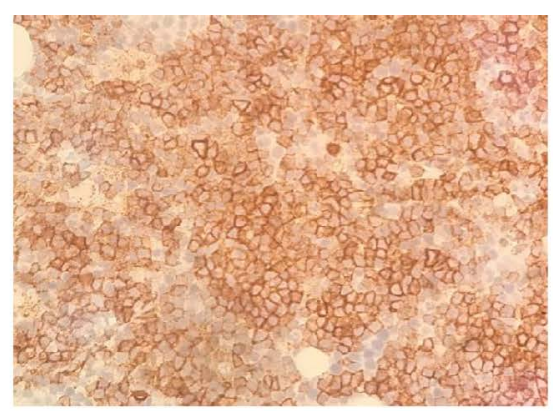

(b)

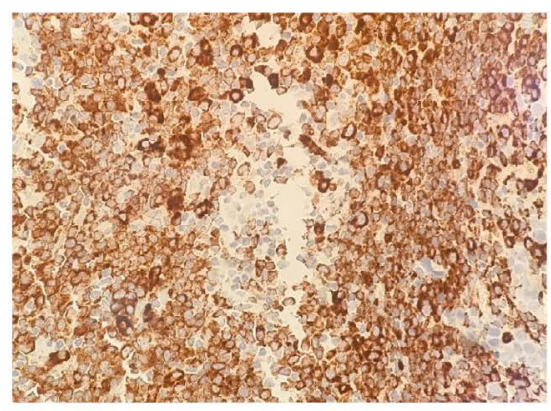

(d)

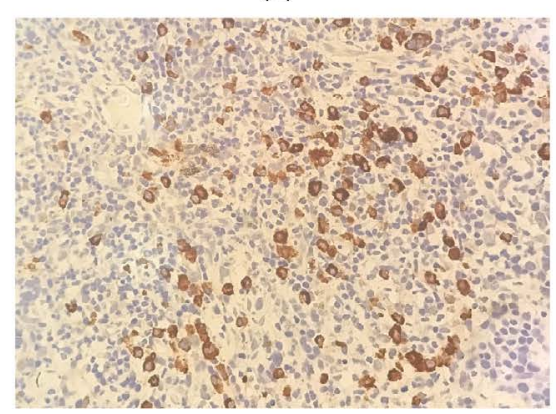

(f)

Figure 3. (a) Trephine biopsy H\&E demonstrates infiltration by clusters of lymphoplasmacytoid cells ((b)-(f)) Panel of immunohistochemical stains (Dako Artisan Link); Neoplastic cells stain positively for CD20, CD138 and VS38C. The neoplastic cells are lambda restricted. 
for CD19, CD20, CD22 and CD38 with a Ki 67 of $40 \%$. They were negative for both CD5 and CD10. Bone marrow flow cytometric analysis showed a cluster of $65 \%$ of abnormal lymphocytes expressing CD19, CD20, CD22, FMC7, CD38, CD 138 and cLambda restriction. Molecular analysis of the bone marrow specimen showed presence of MYD88 L265P gene mutation. Serum protein electrophoresis (SPEP) demonstrated an IgM level of $8 \mathrm{~g} / \mathrm{L}$. The serum free light chains (SFLC) were predominantly lambda of $2350 \mathrm{~g} / \mathrm{L}$ with a lambda kappa ratio (LKR) of 52. The Pan-CT imaging did not demonstrate any lymphadenopathy and organomegaly.

A diagnosis of Waldenstrom macroglobulinemia (IPSSWM: Intermediate risk-age $>65$, hemoglobin $<11.5 \mathrm{~g} / \mathrm{dL}$, platelets $<100 \times 10^{9} / \mathrm{L}$, normal beta-2-microglobulin, IgM $<70 \mathrm{~g} / \mathrm{L}$ ) was established [7]. He was given 2 cycles of R-B chemotherapy but developed worsening anemia requiring blood transfusion albeit improving white cell counts. He was then switched to 6 cycles of Bortezomib-Rituximab-dexamethasone (BRD) immunochemotherapy, in which he responded. He has been in complete remission for the past 16 months with normalization of peripheral blood counts and undetectable levels of IgM and lambda light chains on SPEP and SFLC respectively.

\section{Case Presentation 4}

A 59-year old gentleman of Malay ethnicity with no prior medical illness presented to the hematology department with loss of weight, anorexia, fever and night sweats for the past 10 weeks. He had no significant family history. He was a non-smoker and a teetotaller. He did not consume any traditional or recreational drugs. He worked as a soldier.

Physical examination revealed an alert gentleman. He had an Eastern Co-operative oncology Group) ECOG performance score of 0 (fully active). He had a palpable spleen of $3 \mathrm{~cm}$. There was palpable bilateral inguinal lymphadenopathy. There were no other palpable lymph nodes or organomegaly. Other systemic examinations were unremarkable.

The complete blood count showed a normochromic normocytic anemia of 7.6 $\mathrm{g} / \mathrm{dL}$, total white cell count of $35 \times 10^{9} / \mathrm{L}$ (predominantly lymphocytosis) and a reduced platelet count of $102 \times 10^{9} / \mathrm{L}$. The lactate dehydrogenase level was elevated at $650 \mathrm{U} / \mathrm{L}$. Peripheral blood film (Figure 4(a)) showed numerous abnormal pleomorphic lymphocytes with some being exhibiting prominent nucleoli. The bone marrow aspiration demonstrated lymphocytosis with suppressed trilineage hematopoiesis. The trephine biopsy H \& E (Figure 4(b)) portrayed infiltration by diffuse sheets of blastic looking (blastoid) pleomorphic lymphocytes exhibiting prominent nucleoli. The neoplastic cells (Figures 4(c)-(e)) stained positive for CD19, CD20, cyclin D1 and SOX 11. The bone marrow flow cytometric analysis showed $74 \%$ cluster of lymphocytes expressing CD5, CD19, CD20, CD22 and CD79a. Bone marrow cytogenetic analysis using Giemsa banding technique did not reveal any 17p/TP53 gene deletion. The pan-CT imaging demonstrated multiple lymphadenopathy involving the cervical, axillary, 


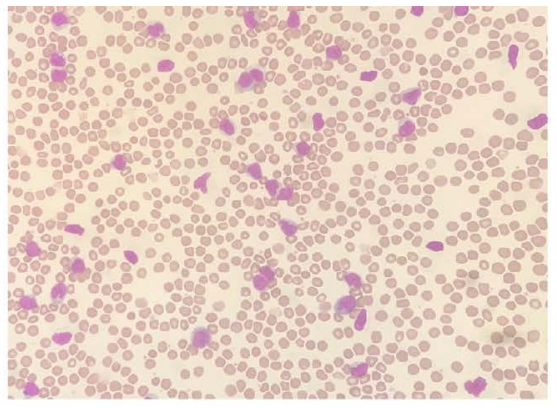

(a)

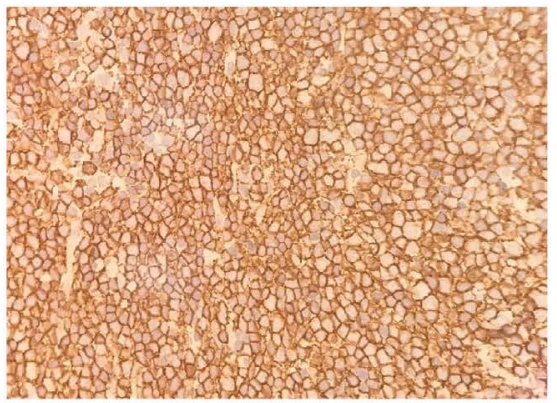

(c)

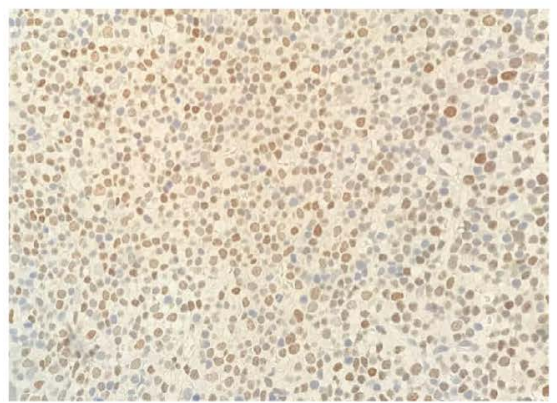

(e)

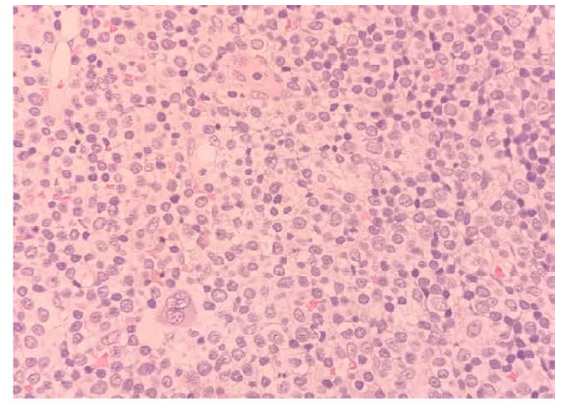

(b)

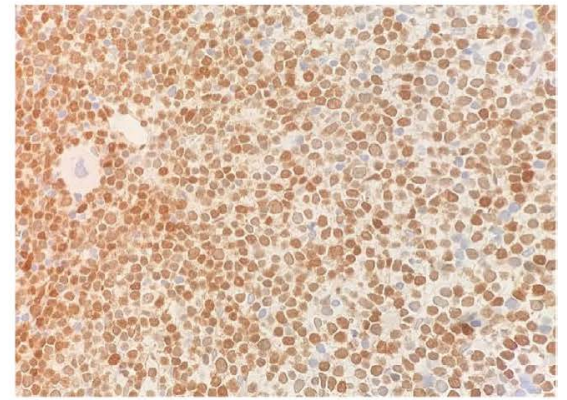

(d)

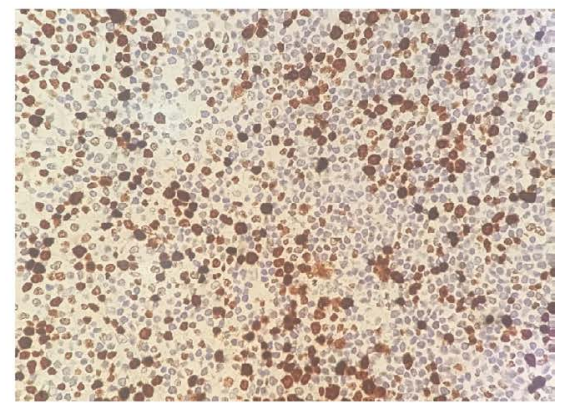

(f)

Figure 4. (a) Peripheral blood film shows numerous abnormal pleomorphic lymphocytes. (b) Trephine biopsy H\&E demonstrated diffuse infiltration by sheets of blastic looking (blastoid) lymphocytes ((c)-(e)) Panel of immunohistochemical staining (Dako Artisan Link): The neoplastic cells stain positive for CD20, Cyclin D1 and SOX11. (f) The Ki 67 is $40 \%$.

intraabdominal and inguinal lymph nodes, the largest measuring $4 \times 4 \mathrm{~cm}$.

A diagnosis of Stage IV leukemic phase mantle cell lymphoma (combined MIPI: Intermediate risk) was established. He was treated using the European mantle cell lymphoma network protocol; 3 cycles of R-CHOP with alternating 3 cycles of R-DHAP (rituximab-dexamethasone, cytarabine, cisplatin). The repeated bone marrow study and 18-FDG PET/CT imaging were in complete remission. He underwent consolidation BEAM (carmustine, etoposide, doxorubicin and melphalan) conditioning followed by autologous stem cell transplantation. Currently, he has been in complete remission for the past 2 years.

\section{Discussion}

Interesting manifestations of low-grade B-cell lymphoproliferative disorders at a 
tertiary referral centre are described.

\subsection{Histomorphology and Immunophenotyping}

Follicular lymphoma (FL) is classified into three main grades based on the population of centroblasts per high power field (hpf); Grade 1:5 or less centroblasts per hpf, 6 - 15 centroblasts per hpf, Grade 3A: >15 centroblasts per hpf and Grade 3B: pure sheets of centroblasts [8]. The patient illustrated here demonstrated $12-13$ centroblasts per hpf microscopy on the cervical lymph node histology, thus, giving rise to the classification of Grade 2 FL (a low-grade FL). Grade $3 \mathrm{FL}$ could be challenging to diagnose on histology. An experienced anatomic pathologist is required to accurately define between Grade 3A and 3B FL. FL rarely involves the peripheral blood (10\% of all cases) unlike other low-grade lymphomas such as MZL, MCL and WM. Lymph node histology demonstrates partial or complete architectural effacement with presence of neoplastic follicles consisting of centrocytes or centroblasts which is similarly seen in this patient'. Follicular dendritic meshwork is usually absent in diffuse areas. Bone marrow trephine biopsy may show paratrabecular neoplastic follicles with or without interstitial infiltration. Neoplastic cells often stain positive for CD19, CD20, CD22, CD79a and BCL-2. Germinal centre markers such as CD10, BCL-6, HGAL and LMO2 are expressed. Grade $3 \mathrm{FL}$ is commonly negative for BCL-2 and germinal centre markers [9]. As opposed to SMZL, FL usually affects the spleen white pulp [9].

This patient in this case presents with an unusual presentation of EMZL of the left pleura as opposed to bilateral pleural involvement as documented in most literature. The other differential diagnoses which should be considered for a unilateral pleural effusion are pleural tuberculosis, infection and lung malignancy. However, they were promptly excluded in this patient on the pleural biopsy.

MZL is characterised by the presence of centrocyte-like lymphocytes which are described as small-to-medium in size exhibiting irregular nuclei, inconspicuous nucleoli and pale cytoplasm on histomorphology [10]. Neoplastic lymphocytes stain positive for CD19, CD20, CD22, CD79a and BCL-2 on immunohistochemistry. They are negative for CD5, CD10, CD23 and BCL-6 [10]. Microbial agents which have been linked with EMZL are Helicobacter pylori (gastric EMZL), Chlamydia psittaci (ocular EMZL), Borrelia Burgdorferi (skin EMZL) and Campylobacter jejuni (small intestine EMZL) which can be detected on tissue immunohistochemistry, in-situ hybridisation (ISH) or PCR. However, there has been no distinct microbial agent associated with pleural EMZL.

Gastric involvement is most common in EMZL. A mandatory oesophagogastroduodenoscopy (OGDS) and if available, an endoscopic ultrasound (EUS) are commonly utilized to evaluate the presence of gastric EMZL and the associated gastric wall and regional perigastric lymph node infiltration. Helicobacter pylori infection, if possible, should be demonstrated on tissue immunohistochemistry. Other alternatives for detection include urea breath analysis or monoclonal stool 
antigen test. PET imaging does not provide any additional information and does not correlate with overall survival in MZL but may be useful to guide clinicians on lymph node sites to biopsy if there is clinical or laboratory suspicion (e.g. elevated lactate dehydrogenase) of transformation to high grade lymphoma [11].

SMZL which affects the splenic red pulp is best diagnosed on spleen histology (splenectomy specimen) but this method requires invasive surgery. Peripheral blood and bone marrow morphology and flow cytometry may be helpful to diagnose SMZL. SMZL may or may not demonstrate classic polar villi lymphocytes. Bone marrow histomorphology in SMZL often share similar features with splenic diffuse small cell red pulp lymphoma, hence the role of a splenectomy specimen becomes important [12].

WM is diagnosed by the presence of both lymphoplasmacytic cells and monoclonal IgM on serum electrophoresis and immunofixation studies as described in this patient. The IgM levels are usually $>30 \mathrm{~g} / \mathrm{L}$ in WM but can be of any concentration. Neoplastic cells express CD19, CD20, CD22, CD38, CD79a, FMC7 and CD138 [13]. They do not express CD5, CD10 or CD23. WM usually do not exhibit osteolytic lesion, renal dysfunction or hypercalcemia. Patients with chronic hepatitis $\mathrm{C}$ infection should have serum cryoglobulin assessed to exclude cryoglobulinemia. Whole-body CT should be performed on all patients considered for therapy to assess presence of lymphadenopathy. PET imaging does not offer additional information but may be useful if there is clinical or laboratory evidence of transformation.

MCL is usually characterised by the presence of small-to-medium abnormal lymphocytes with irregular nuclei. Neoplastic lymphocytes may resemble small mature looking B lymphocytes (CLL-like), MZL-like lymphocytes, blastoid or pleomorphic-type on histomorphology. The neoplastic cells express CD5, CD19, CD20, CD22 and cyclin D1. They do not usually express CD10. Cyclin D1 overexpression is seen in $>90 \%$ of MCL. Those who do not express Cyclin D1 should be assessed for the presence of SOX11.

\subsection{Molecular and Cytogenetic Expression}

Molecular and gene expression profiling of tumour tissue may play a role in risk stratification in low grade lymphomas. The molecular mutations (m7-FLIPI) recognised in FL are EZH2, ARID1A, CREBBP, CARD11, FOXO1, EP300 and MEF2B [14]. However, this molecular profiling was not performed in this patient due to financial constraints. This clinicogenetic risk model which includes the m7-FLIPI, FLIPI and ECOG performance status improves the risk stratification model in advanced stage FL receiving rituximab based immunochemotherapy [14]. However, m7-FLIPI is not prognostic in FL patients treated with first line rituximab without chemotherapy, hence suggesting prognostic value is dependent on therapeutic regimen [15].

There are no specific molecular or genetic abnormalities encountered in pleural EMZL. It is interesting to note that a proportion of gastric EMZL exhibit 
$\mathrm{t}(11 ; 18)$ cytogenetic abnormality on tissue fluorescent in-situ hybridisation (FISH) cytogenetic analysis which may explain poor response to triple eradication therapy [16]. Molecular and cytogenetic abnormalities frequently encountered in NMZL include IgH rearrangements, trisomy 3, trisomy 18, 1q21 and 1q34 abnormalities which may explain its poorer prognosis as compared to other subtypes of MZL [16].

Molecular analysis of bone marrow sample using a sensitive method, such as allele-specific oligonucleotide PCR or next generation sequencing (NGS) demonstrate the presence of MYD88 L265P gene mutation in approximately $90 \%$ of patients [17]. Chemokine receptor type-4 (CXCR4) is seen in $30 \%$ of WM which contributes to disease progression and treatment failure [17]. WM patients who do not demonstrate the classic MYD88 L265P mutation may have other MYD88 mutations including wild-type. Molecular testing for CXCR4 is not recommended in routine practise as there is currently no specific targeted therapy available. MYD88 may also be seen infrequently in other lymphomas such as MZL. Other cytogenetic abnormalities encountered in WM/LPL are trisomy 18, $13 q$ deletion, $17 \mathrm{p}$ deletion, trisomy 4 and 11q deletions [17].

High risk genetic aberrations such as TP53/17p deletion, CDKN2A deletion and NOTCH1 mutations are associated with a poorer outcome in MCL.

\subsection{Treatment Modalities}

Management of low-grade lymphoma has been centred around anti CD20, rituximab-based therapy which has emerged as the mainstay of therapy. Watchful waiting may be appropriate in asymptomatic non-bulky limited and advanced stage FL. Symptomatic non-bulky limited-stage (Non-Grade 3B) FL (Stage I-II) may benefit from involved site radiotherapy (ISRT) 24 Gray which is potentially curable [18]. However, in patients with symptomatic Stage I-II with large tumour burden or advanced disease (Stage III-IV), the preferred treatment is anti-CD20 rituximab in combination with chemotherapy. First line therapies such as $\mathrm{R}-\mathrm{CHOP}, \mathrm{R}-\mathrm{CVP}$ and $\mathrm{R}-\mathrm{B}$ have demonstrated an overall response rate (ORR) of $96 \%, 81 \%$ and $93 \%$ respectively [19]. Obinutuzumab in combination with chemotherapy (G-CVP, G-CHOP or G-bendamustine) as first line therapy followed by obinutuzumab 2-monthly maintenance up to 2 years in advanced stage FL has demonstrated a longer PFS and higher MRD negativity (no difference in overall survival) as compared to Rituximab based chemotherapy [20]. However, increased Grade 3 adverse events such as neutropenia and infections were seen in the Obinutuzumab based chemotherapy group. PET-CT imaging is now the standard of care in FL. Positive PET at the end of treatment predicts early disease progression and risk of death. Radiotherapy may be considered in PET positive areas. MRD analysis of peripheral blood using NGS testing may identify patients with residual disease. Patients with PET and MRD positivity after induction therapy may have radioimmunotherapy added to rituximab maintenance. Anthracycline based chemotherapy such as $\mathrm{R}-\mathrm{CHOP}$ is the preferred regime for 
Grade 3B FL or presence of clinical features of large cell transformation. Rituximab maintenance every two months for up to two years have shown increased PFS and OS in advanced stage FL. Rituximab maintenance has showed superiority to radioimmunotherapy as maintenance in advanced stage FL [21]. Previously untreated elderly FL not suitable for conventional chemotherapy may benefit from rituximab-chlorambucil combination therapy or radioimmunotherapy (yttrium-ibritumomab-tiuxetan)-zevalin alone. Radioimmunotherapy, purine analogue based therapy (Fludarabine, cyclophosphamide and rituximab), platinum salts, PI3-K inhibitors (idelalisib or copanlisib) are options in relapsed disease. If the relapse is more than 12 - 24 months, the same treatment regime could be re-used. Rituximab should be added in relapsed disease if the patient has achieved remission for more than 6 - 12 months. High dose therapy-ASCT prolongs PFS and OS in relapsed disease, but not as frontline therapy in FL [22].

Triple eradication therapy combining a proton pump inhibitor for 4 weeks, clarithromycin $500 \mathrm{mg}$ twice daily and either amoxycillin $1 \mathrm{~g}$ daily or metronidazole $400 \mathrm{mg}$ thrice daily for 10 - 14 days is the mainstay of treatment in both $\mathrm{H}$ pylori positive and negative gastric EMZL [23]. False negative results are known to occur in $\mathrm{H}$ pylori negative gastric EMZL as other species of Helicobacter may be present. Response to therapy could be assessed through a urea breath test not earlier than 6 weeks. An endoscopy is usually performed at $6-12$ months to assess remission and regression of lymphoma in $\mathrm{H}$ pylori gastric EMZL [24]. It may take a few weeks to a year to achieve remission with triple eradication therapy. For $\mathrm{H}$ pylori negative gastric EMZL, a repeat endoscopy should be performed earlier at 3 - 6 months and should there be no signs of regression of lymphoma, radiotherapy should be considered. Once endoscopic remission is achieved, repeat endoscopy with biopsy should be performed every 6 months for up to 2 years as gastric EMZL predisposes the risk to gastric adenocarcinoma. Involved site radiotherapy (ISRT) of 24 - 30 Gy over 3 - 4 weeks is effective as second line in patients who do not respond to $\mathrm{H}$ pylori eradication therapy [25]. Gastric EMZL expressing $t(11 ; 18)$ shows good response to 4 cycles of rituximab-bendamustine combination therapy in a phase 2 clinical trial [26]. Radiotherapy is also effective as first line therapy in other localised EMZL. Watch and wait approach is frequently applied in asymptomatic nodal and splenic MZL including advanced disease. Rituximab based therapy such as rituximab-cyclophosphamide, vincristine, prednisolone (R-CVP), rituximab, cyclophosphamide, vincristine, prednisolone (R-CHOP) or R-B (rituximab-bendamustine) are employed as first-line therapy for symptomatic nodal MZL [26]. The use of rituximab maintenance in advanced NMZL is optional as there are no prospective studies to support this practise.

Traditional splenectomy has largely been replaced with rituximab monotherapy in SMZL. Splenectomy has shown good 5-year OS of $70 \%-80 \%$ but it is an invasive major procedure not without complications. Rituximab monotherapy $375 \mathrm{mg} / \mathrm{m}^{2}$ weekly for $4-8$ weeks has shown an overall response rate (ORR) of more than $80 \%$ [27]. Patients who do not respond to rituximab monotherapy 
may benefit from combination rituximab-chemotherapy regimens such as R-CVP/R-CHOP or splenectomy.

International prognostic scoring system for Waldenstrom macroglobulinemia (IPSSWM) was developed by Dr Pierre Morel of France in year 2007 and it serves as a predictive model to determine long term outcome in symptomatic patients requiring treatment. Some authors have proposed revised scoring systems since treatments for WM have evolved. Watchful waiting is used for asymptomatic WM. Anti-CD20 monoclonal antibody (rituximab) based immunochemotherapy is the mainstay of treatment in WM [28]. Six cycles of rituximab in combination with cyclophosphamide and dexamethasone (RCD) has demonstrated an overall survival of 8-years [29]. Rituximab-bendamustine (R-B) combination therapy is useful for patients presenting with high disease burden [29]. There has not been any randomised controlled trials comparing RCD and R-B. Other regimens include proteasome inhibitor such bortezomib monotherapy or combination bortezomib with rituximab and dexamethasone (BRD) therapy. Five cycles of BRD has shown an overall survival of $66 \%$ at 7 years [30]. Bortezomib based combination regimens and ibrutinib monotherapy are considered in patients presenting with high IgM levels and hyperviscosity [30]. Elderly patients unable to tolerate chemotherapy may benefit from ibrutinib monotherapy.

Treatment for symptomatic mantle cell lymphoma of any stage is usually classified into three main categories; young $(<65)$, elderly 65 years old and over, and compromised individuals. Radiotherapy for limited stage disease is associated with relapse, thus requiring a sequential approach of chemotherapy followed by consolidation radiotherapy [31]. This patient who was a fit 59-year-old was treated with the European mantle cell lymphoma network protocol consisting of alternating cycles of R-CHOP and R-DHAP polychemotherapy. Rituximab and high dose cytarabine based polychemotherapy regimens such as R-CHOP/R-DHAP and Nordic protocol such as R-maxi-CHOP/R-HiDAC (alternating cycles of rituximab, maximal doses of cyclophosphamide, doxorubicin, vincristine and prednisolone with high dose cytarabine) remain the therapy of choice in younger fit patients below the age of 65 . Young fit patients treated with R-hyperCVAD (cyclophosphamide, doxorubicin, vincristine, prednisolone alternating with methotrexate and cytarabine) frequently achieve very good overall response rates. Intensive induction chemotherapy regimens as mentioned above followed by consolidation autologous SCT and maintenance rituximab for a duration of 2 years is associated with a better overall survival [32]. Fit elderly patients over the age of 65 may benefit from less intensive chemotherapy regimens including R-B, bortezomib based chemotherapy (bortezomib, rituximab-cyclophosphamide, doxorubicin, prednisolone), R-CHOP and R-BAC (rituximab, bendamustine, doxorubicin, cytarabine). Elderly compromised/frail individuals are usually treated with metronomic oral chemotherapy such chlorambucil or PEP-C (prednisolone, etoposide, procarbazine, cyclophosphamide). High risk cytogenetic mutations including 17p/TP53 mutated MCL do not benefit from standard 
regimens and thus, may require novel agents such as ibrutinib and venetoclax as frontline therapy [33]. It is interesting to note a negative PET-CT at end of treatment and MRD negativity of peripheral blood by the means of NGS have become important independent prognostic indicators in MCL but they has yet to demonstrate an improvement in OS [34]. If relapses occur early (within 12 months), a different regime should be used. Rituximab can be re-used if the last dose was not less than 6 months [35]. A variety of treatments used in the relapsed setting include ibrutinib monotherapy, venetoclax monotherapy, combined ibrutinib-venetoclax therapy, combined rituximab-lenalidomide therapy, temsirolimus and bortezomib. Temsirolimus and bortezomib should be used in combination with chemotherapy. Ibrutinib and venetoclax have been associated with high response rates in relapsed MCL.

\section{Conclusion}

Low-grade lymphomas are indolent but most often incurable and are noted for their frequent relapses. There are many novel agents available currently which are often tolerable to treat relapse disease including in the elderly. PET-CT imaging and MRD analysis of the peripheral blood show promise as useful prognostic indicators in some low-grade lymphomas.

\section{Acknowledgements}

None.

\section{Consent}

Written informed consent was obtained from the patients for publication of this case series and accompanying images. A copy of the written consent is available for review by the Editor-in-Chief of this journal on request.

\section{Author Contributions}

G. K. analysed the data, designed the paper, contributed to the writing of the manuscript, made critical revisions and approved the final manuscript.

\section{Conflicts of Interest}

The author declares no potential conflicts of interests.

\section{Ethical Approval}

Ethical approval is not required as this is not a clinical trial.

\section{Funding}

Self-funding.

\section{Guarantor}

Ganesh Kasinathan is the guarantor of this manuscript. 


\section{References}

[1] Swerdlow, S.H., Campo, E., Pileri, S.A., et al. (2016) The 2016 Revision of the World Health Organisation Classification of Lymphoid Neoplasms. Blood, 127, 2375-2390. https://doi.org/10.1182/blood-2016-01-643569

[2] Fischer, T., Zing, N.P., Chiattone, C.S., Federico, M. and Luminari, S. (2018) Transformed Follicular Lymphoma. Annals of Hematology, 97, 17-29.

https://doi.org/10.1007/s00277-017-3151-2

[3] Link, B.K. (2018) Transformation of Follicular Lymphoma-Why Does It Happen, and Can It Be Prevented? Best Practise \& Research. Clinical Hematology, 31, 49-56. https://doi.org/10.1016/j.beha.2017.10.005

[4] Sriskandarajah, P. and Dearden, C.E. (2017) Epidemiology and Environmental Aspects of Marginal Zone Lymphomas. Best Practise \& Research. Clinical Hematology, 30, 84-91. https://doi.org/10.1016/j.beha.2016.07.002

[5] Thieblemont, C., Molina, T. and Davi, F. (2016) Optimizing Therapy for Nodal Marginal Zone Lymphoma. Blood, 127, 2064-2071. https://doi.org/10.1182/blood-2015-12-624296

[6] Xochelli, A., Kalpadakis, C., Gardiner, A., et al. (2014) Clonal B-Cell Lymphocytosis Exhibiting Immunophenotypic Features Consistent with a Marginal-Zone Origin: Is This a Distict Entity? Blood, 123, 1199-1206. https://doi.org/10.1182/blood-2013-07-515155

[7] Paiva, B., Corchete, L.A., Vidriales, M.B., et al. (2015) The Cellular Origin and Malignant Transformation of Waldenstrom Macroglobulinemia. Blood, 125, 2370-2380. https://doi.org/10.1182/blood-2014-09-602565

[8] Ott, G., Katzenberger, T., Lohr, A., et al. (2002) Cytomorphologic, Immunohistochemical, and Cytogenetic Profiles of Follicular Lymphoma: 2 Types of Follicular Lymphomagrade 3. Blood, 99, 3806-3812. https://doi.org/10.1182/blood.V99.10.3806

[9] Takata, K., et al. (2014) Pathology of Follicular Lymphoma. J Clin Exp Hematop, 54, 3-9.

[10] Salama, M.E., Lossos, I.S. and Warnke, R.A. (2009) Immunoarchitectural Patterns in Nodal Marginal Zone B-Cell Lymphoma: A Study of 51 Cases. American Journal of Clinical Pathology, 132, 39-49. https://doi.org/10.1309/AJCPZQ1GXBBNG8OG

[11] Vaxman, I., Bernstine, H., Kleinstern, G., Hendin, N., Shimony, S., Domachevsky, L., Gurion, R., Groshar, D., Raanani, P. and Gafter-Gvili, A. (2019) FDG PET/CT as a Diagnostic and Prognostic Tool for the Evaluation of Marginal Zone Lymphoma. Hematological Oncology, 37, 168-175. https://doi.org/10.1002/hon.2578

[12] Ponzoni, M., Kanellis, G., Pouliou E, et al. (2012) Bone Marrow Histopathology in the Diagnostic Evaluation of Splenic Marginal Zone and Splenic Diffuse Red Pulp Small B-Cell Lymphoma: A Reliable Substitute for Spleen Histopathology? The American Journal of Surgical Pathology, 36, 1609-1618.

[13] Owen, R.G., Treon, S.P., Al-Katib, A., et al. (2003) Clinicopathological Definition of Waldenstrom Macroglobulinemia: Consensus Pabnel Recommendations from the Second International Workshop on Waldenstrom Macroglobulinemia. Seminars in Oncology, 30, 110-115.

[14] Pastore, A., Jurinovic, V., Kridel, R., et al. (2015) Integration of Gene Mutations in Risk Prognostification for Patients Receiving First-Line Immunochemotherapy for Follicular Lymphoma: A Retrospective Analysis of a Prospective Clinical Trial and Validation in a Population-Based Registry. The Lancet Oncology, 16, 1111-1122. 
https://doi.org/10.1016/S1470-2045(15)00169-2

[15] Lockmer, S., Ren, W.C., Brodtkorb, M., Østenstad, B., Wahlin, B.E. Pan-Hammarström, Q. and Kimby, E. (2020) M7-FLIPI Is Not Prognostic in Follicular Lymphoma Patients with First-Line Rituximab Chemo Free Therapy. British Journal of Haematology, 188, 259-267. https://doi.org/10.1111/bjh.16159

[16] Zucca, E. and Bertoni, F. (2016) The Spectrum of MALT Lymphoma at Different Sites: Biological and Therapeutic Relevance. Blood, 127, 2082-2092. https://doi.org/10.1182/blood-2015-12-624304

[17] Treon, S.P., Xu, L., and Hunter, Z. (2015) MYD88 L265P Mutations and Response to Ibrutinib in Waldenstrom Macroglobulinemia. The New England Journal of Medicine, 373, 584-586. https://doi.org/10.1056/NEJMc1506192

[18] Hoskin, P.J., Kirkwood, A.A., Popova, B., et al. (2014) 4 Gy versus 24 Gy Radiotherapy for Patients with Indolent Lymphoma (FORT): A Randomised Phase 3 Non-Inferiority Trial. The Lancet Oncology, 15, 457-463. https://doi.org/10.1016/S1470-2045(14)70036-1

[19] Marcus, R., Imrie, K., Solal-Celogny, P., Catalano, J.V., Dmoszynska, A., Raposo, J.C., Offner, F.C., Gomez-Codina, J., Belch, A., Cunningham, D., Wassner-Fritsch, E. and Stein, G. (2008) Phase III Study of R-CVP Compared with Cyclophosphamide, Vincristine, and Prednisolone Alone in Patients with Previous Untreated Advanced Follicular Lymphoma. Journal of Clinical Oncology, 26, 4579-4586. https://doi.org/10.1200/JCO.2007.13.5376

[20] Pott, C., Hoster, E., Kehden, B., et al. (2016) Minimal Residual Disease in Patients with Follicular Lymphoma Treated with Obinutuzumab or Rituximab as First-Line Induction Immunochemotherapy and Maintenance in the Phase 3 GALLIUM Study. Blood, 128, 613. https://doi.org/10.1182/blood.V128.22.613.613

[21] Lopez-Guillermo, A., Canales, M.A., Dlouhy, I., et al. (2013) A Randomised Phase II Study Comparing Consolidation with a Single Dose of 90-Yttrium-IbritumomabTiutexan (Zevalin) vs Maintenance with Rituximab Flor Two Years in Patients with Newly Diagnosed Follicular Lymphoma (FL) Responding to R-CHOP. Preliminary Results at 36 Months from Randomisation. Blood, 122, 369. https://doi.org/10.1182/blood.V122.21.369.369

[22] Montoto, S., Corradini, P., Dreyling, M., Ghielmini, M., Kimby, E., López-Guillermo, A., Mackinnon, S., Marcus, R.E., Salles, G., Schouten, H.C., Sureda, A. and Dreger, P. (2013) Indications for Hematopoietic Stem Cell Transplantation in Patients with Follicular Lymphoma: A Consensus Project of the EBMT-Lymphoma Working Party. Haematologica, 98, 1014-1021. https://doi.org/10.3324/haematol.2013.084723

[23] Zullo, A., Hassan, C., Cristofari, F., et al. (2013) Effects of Helicobacter Pylori-Negative, Gastric Low-Grade Mucosa Associated Lymphoid Tissue Lymphoma Patients: A Systematic Review. Journal of Clinical Gastroenterology, 47, 824-827.

[24] Ruskone-Fourmestraux, A., Fischbach, W., Aleman, B.M., Boot, H., Du, M.Q., Megraud, F., Montalban, C., Raderer, M., Savio, A. and Wotherspon, A. (2011) EGILS Consensus Report. Gastric Extranodal Marginal Zone B-Cell Lymphoma of MALT. Gut, 60, 747-758. https://doi.org/10.1136/gut.2010.224949

[25] Wirth, A., Gospodarowicz, M., Pintilie, M., et al. (2013) Long-Term Outcome for Gastric Marginal Zone Lymphoma Treated with Radiotherapy: A Retrospective Muti-Center, International Extranodal Lymphoma Study Group Study. Annals of Oncology, 24, 1344-1351. https://doi.org/10.1093/annonc/mds623

[26] Salar, A., Domingo-Domenech, E., Panizo, C., et al. (2017) Long-Term Results of a Phase II Study of Rituximab and Bendamustine for Mucosa Associated Lymphoid 
Tissue Lymphoma. Blood, 130, 1772-1774.

https://doi.org/10.1182/blood-2017-07-795302

[27] Kalpadakis, C., Pangalis, G.A., Sachanas, S., et al. (2018) Rituximab Monotherapy in Splenic Marginal Zone Lymphoma: Prolonged Responses and Potential Benefit from Maintenance. Blood, 132, 666-670. https://doi.org/10.1182/blood-2018-02-833608

[28] Dimopoulas, M.A., Zervas, C., Zomas, A., et al. (2002) Treatment of Waldenstrom Macroglobulinemia with Rituximab. Journal of Clinical Oncology, 20, 2327-2333. https://doi.org/10.1200/JCO.2002.09.039

[29] Kastritis, E., Gavriatopoulou, M., Kyrtsonis, M.C., et al. (2015) Dexamethasone, Rituximab and Cyclophosphamide as Primary Treatment of Waldenstrom Macroglobulinemia: Final Analysis of a Phase 2 Study. Blood, 126, 1392-1394.

https://doi.org/10.1182/blood-2015-05-647420

[30] Gavriatopoulou, M., Garcia-Sanz, R., Kastritis, E., et al. (2017) BDR in Newly Diagnosed Patients with WM: Final Analysis of a Phase 2 Study after a Minimum Follow-Up of 6 Yrars. Blood, 129, 456-459.

https://doi.org/10.1182/blood-2016-09-742411

[31] Leitch, H.A., Gascoyne, R.D., Chhanabhai, M., et al. (2003) Limited-Stage Mantle Cell Lymphoma. Annals of Oncology, 14, 1555-1561. https://doi.org/10.1093/annonc/mdg414

[32] Hermine, O., Hoster, E., Walewski, J., et al. (2016) Addition of High-Dose Cytarabine to Immunochemotherapy Before Autologous Stem-Cell Transplantation in Patients Aged 65 Years or Younger with Mantle Cell Lymphoma: A Randomised, Open Label, Phase 3 Trial of the European Mantle Cell Lymphoma Network. The Lancet, 388, 565-575. https://doi.org/10.1016/S0140-6736(16)00739-X

[33] Woyach, J.A., Furman, R.R., Liu, T.-M., et al. (2014) Resistance Mechanisms for the Bruton's Tyrosine Kinase Inhibitor Ibrutinib. The New England Journal of Medicine, 370, 2286-2294. https://doi.org/10.1056/NEJMoa1400029

[34] Bailly, C., Carlier, T., Touzeau, C., Arlicot, N., Kraeber-Bodéré, F., Le Gouill, S. and Bodet-Milin, C. (2019) Interest of FDG-PET in the Management of Mantle Cell Lymphoma. Frontiers of Medicin, 6, 70. https://doi.org/10.3389/fmed.2019.00070

[35] Visco, C., Chiappella, A., Nassi, L., et al. (2017) Rituximab, Bendamustine, and Low Dose Cytarabine as Induction Therapy in Elderly Patients with Mantle Cell Lymphoma: A Multicentre, Phase 2 Trial from Fondazion Italiana Linfomi. The Lancet Haematology, 4, E15-E23. https://doi.org/10.1016/S2352-3026(16)30185-5 